\title{
Avaliação de espécies arbóreas para composição de cortinas de segurança contra incêndios florestais
}

\author{
Bruna KOVALSYKI ${ }^{1}$, Luiz Donizeti CASIMIRO JUNIOR ${ }^{1}$, Rafaela ASSUNÇÃO ${ }^{1}$, \\ Raquel Costa Chiao TRAVENISK ${ }^{2}$, Alexandre França TETTO ${ }^{1}$, Antonio Carlos BATISTA ${ }^{1}$ \\ ${ }^{1}$ Departamento de Ciências Florestais, Universidade Federal do Paraná, Curitiba, PR, Brasil \\ ${ }^{2}$ Secretaria Municipal de Saneamento e Meio Ambiente, Sarandi, PR, Brasil \\ *E-mail: kovalsyki.b@gmail.com
}

Recebido em março/2018; Aceito em outubro/2018.

\begin{abstract}
RESUMO: Cortina de segurança é um plantio em faixas com espécies vegetais de menor combustibilidade do que as do cultivo principal, a fim de reduzir ou evitar a propagação do fogo. O presente trabalho teve por objetivo avaliar o potencial das espécies Magnolia grandiflora L., Michelia champaca L., Viburnum odoratissimum Ker Gawl., Casearia sylvestris Swartz e Bougainvillea glabra Choisy para compor cortinas de segurança. Para isso, as espécies foram analisadas em função do teor de umidade, altura de chama, velocidade de propagação do fogo, quantidade de material combustível consumido em queimas experimentais e intensidade do fogo. Foram realizadas 10 repetições por espécie e as queimas foram realizadas no Laboratório de Incêndios Florestais, do Departamento de Ciências Florestais, da Universidade Federal do Paraná, em parcelas de $1 \mathrm{~m}^{2}$, com uma carga de $1 \mathrm{~kg}$ de material combustível fino seco em estufa. As espécies que se destacaram nas análises foram B. glabra e C. sylvestris, as quais indicaram baixa combustibilidade, apresentando assim potencial para uso em cortina de segurança na prevenção de incêndios florestais.

Palavras-chave: barreiras verdes, prevenção de incêndios, altura de chama, proteção florestal.
\end{abstract}

\section{Evaluation of tree species for the composition of green firebreaks against forest fires}

\begin{abstract}
Green firebreaks are vegetation stripes with a lower combustibility than that of the main crop and are intended to prevent or restrict the spread of fire. The present study aimed to evaluate the potential of the following tree species to compose firebreaks: Magnolia grandiflora L., Michelia champaca L., Viburnum odoratissimum Ker Gawl., Casearia sylvestris Swartz and Bougainvillea glabra Choisy. The species were analyzed according to their moisture content, flame height, fire spread rate, amount of fuel consumed in controlled burns, and fire intensity. There were ten repetitions per species and the burns were done at the wildfire laboratory, from the forest sciences department, of Universidade Federal do Paraná, on experimental plots of 1 $\mathrm{m}^{2}$, with $1 \mathrm{~kg}$ of thin fuel load, kiln-dried at $75^{\circ} \mathrm{C}$ for 48 hours. The most notable species from the analyses were B. glabra and C. sylvestris, which indicated low combustibility, showing potential to compose green firebreaks and prevent forest fires.
\end{abstract}

Keywords: green firebreaks, fire prevention, flame height, forest protection.

\section{INTRODUÇÃO}

O fenômeno dos incêndios florestais está mudando, visto que o ambiente florestal e suas interações com o clima e a sociedade têm novas conotações (BOVIO et al., 2017). Bowman et al. (2011) apontam que os humanos modernos podem alterar a atividade natural do fogo, uma vez que modificam os padrões de ignição e ativam os incêndios, causam transformações substanciais no ecossistema e perda de biodiversidade. Segundo esses autores, alguns desses regimes de fogo contemporâneos causam perturbações econômicas devido aos danos provocados às infraestruturas, à degradação dos serviços ecossistêmicos, às ameaças e perdas de vidas e aos efeitos a saúde.

Seidl et al. (2017), em estudo sobre distúrbios florestais sob mudanças climáticas, sugerem que, para condições futuras mais quentes e mais secas, haverá um aumento dos incêndios florestais. Ho et al. (2017), ao considerarem as alterações climáticas, apontam uma elevação no risco de incêndios no estado de Santa Catarina até o final do século, elevando as chances de ocorrências de incêndios, sendo o mesmo esperado para o estado do Paraná (BATISTA et al., 2014).

A ocorrência e frequência dos incêndios florestais estão intimamente relacionados às condições meteorológicas. Juntamente a este fator, a propagação do fogo é influenciada pela topografia, pelo material combustível e seu teor de umidade. A ação de cada um destes fatores é diferente para cada região e para cada época do ano, sendo que os maiores e mais destrutivos incêndios ocorreram sob combinações ideais de material combustível e condições meteorológicas (SOARES et al., 2017).

Com o objetivo de minimizar a incidência e a magnitude dos incêndios, métodos de prevenção e combate vêm sendo desenvolvidos e atualizados. Uma das medidas de prevenção aos incêndios florestais, proposta pela silvicultura preventiva, é a implantação de cortinas de segurança, também conhecida como aceiros, barreiras ou cinturões verdes. Trata-se de um plantio em faixas com espécies de menor inflamabilidade do que as do cultivo principal, com o objetivo de reduzir ou evitar 
a propagação do fogo (HALTENHOFF, 2006). Quando compostos por espécies nativas, estas estruturas podem proporcionar benefícios à biodiversidade, como o fornecimento de alimentos, habitat e oportunidades de dispersão para a fauna (CURRAN et al., 2017).

Esta técnica tem sido amplamente utilizada na região oeste dos Estados Unidos (CORDELL, 2017), Nova Zelândia, China, Indonésia, África Ocidental, Peru e Europa, entretanto, apesar de seu uso extensivo, há lacunas de conhecimento que precisam ser abordados (CURRAN et al., 2017).

No Brasil, apesar de se utilizar faixas de vegetação para algumas finalidades na área rural, como quebra-vento (e.g. Grevillea robusta e Hibiscus sp) (MAPA, 2016), especificamente para a prevenção de incêndios florestais essa prática é incipiente. Estudos sobre inflamabilidade vem sendo realizados com espécies ornamentais, por Batista; Biondi (2009), Batista et al. (2012), Biondi et al. (2014), Kovalsyki et al, (2016).

Batista; Biondi (2009) indicam que Ligustrum lucidum (alfeneiro) possui baixa inflamabilidade em condições reduzidas de umidade, sendo, portanto, uma espécie potencial para uso em cortinas de segurança, muito embora tenha como fator restritivo o fato de ser uma espécie exótica invasora. Outras espécies que possuem inflamabilidade moderada ou baixa são Olea europaea (oliveira) (região do Mediterrâneo) (VÉLEZ, 2009), Camelia sinensis (chá-preto) (China) (TIAN et al., 2007), Coprosma robusta (karamu) (Nova Zelândia) (SCION, 2018).

Uma das maiores dificuldades em implantar cortinas de segurança é a identificação de espécies adequadas para formação dessas estruturas (BATISTA; BIONDI, 2009). Tolerância à seca e folhas perenes são consideradas características importantes das plantas selecionadas para esta finalidade, além do alto teor de umidade e baixos níveis de resinas ou óleos voláteis (WHITE; ZIPPERER, 2010). Para determinação de tais espécies avalia-se, além da inflamabilidade, o comportamento do fogo, que possibilita compreender os principais fatores que incidem sobre o início e a propagação do fogo, assim como no combate dos incêndios.

Considerando-se as características citadas, foram selecionadas cinco espécies arbóreas para este experimento, a fim de avaliar por meio de queimas controladas o comportamento do fogo destas espécies e assim indicar suas potencialidades para uso na implantação de cortinas de segurança.

\section{MATERIAL E MÉTODOS}

Todos os experimentos e análises necessários para o desenvolvimento da pesquisa foram realizados nas dependências do Laboratório de Incêndios Florestais, do Departamento de Ciências Florestais, da Universidade Federal do Paraná.

\subsection{Espécies avaliadas}

As espécies arbóreas analisadas foram: Magnolia grandiflora L. (magnólia-branca), Michelia champaca L. (magnólia-amarela), Viburnum odoratissimum Ker Gawl. (viburno-perfumado), Casearia sylvestris Swartz (cafezeirodo-mato) e Bougainvillea glabra Choisy. (buganvile). Nesta pesquisa utilizou-se a espécie Pinus taeda L. como testemunha para fins comparativos, uma vez que esta espécie é considerada altamente inflamável e tem sido muito cultivada na região sul do Brasil. As espécies selecionadas são descritas a seguir.

Magnolia grandiflora L. (Magnoliaceae): originária dos Estados Unidos atinge até $15 \mathrm{~m}$ de altura. É uma espécie longeva (de crescimento lento), perene e com folhas coriáceas aglomeradas nas extremidades dos ramos, formando copa piramidal na juventude e aberta no envelhecimento da planta (LORENZI et al., 2003). De acordo com Wade (2012), em estudo realizado no estado da Georgia - EUA, M. grandiflora apresentou crescimento moderadamente rápido, resistente ao frio e amplamente adaptável a uma variedade de solos. Além disso, segundo o autor, apresenta poucos problemas de pragas e pode ser cultivada em sol ou sombra. Gilman; Watson (2011a) apontam o uso da magnólia também como quebravento. A espécie é amplamente utilizada no paisagismo em geral, é resistente a danos causados por dióxido de enxofre e suas folhas, frutos, cascas e madeira produzem uma variedade de extratos com potenciais aplicações como produtos farmacêuticos (OUTCALT, 1990).

Michelia champaca L. (Magnoliaceae): árvore originária da Índia e Himalaia, perenifólia e com até $10 \mathrm{~m}$ de altura. Apresenta ramagem disposta de maneira que forma uma copa característica piramidal na juventude (LORENZI et al., 2003). De acordo com Bianchetti et al. (2000), no Brasil a espécie é usada para arborização e para silvicultura emergente, enquanto sua madeira é utilizada para lenha e serraria. Oliveira et al. (2013), em estudo realizado em Uberlândia - MG, indicaram que as sementes ariladas de $M$. champaca, devido a presença de frutos maduros durante quase todo 0 ano, podem representar uma importante fonte alternativa de recursos alimentares para aves, principalmente em período de escassez de frutos nativos.

Viburnum odoratissimum Ker Gawl. (Caprifoliaceae): originária da Índia, China e Japão, atinge até $7 \mathrm{~m}$ de altura. É uma árvore perenifólia, com ramagem densa formando copa arredondada com folhas coriáceas, muito rústica e tolerante às condições adversas de solo e clima, porém tem crescimento lento (LORENZI et al., 2003). De acordo com Gilman; Watson (2011b), esta espécie não apresenta potencial invasivo, sendo tolerante à seca e resistente a pragas.

Casearia sylvestris SW. (Salicaceae, antiga Flacourtiaceae): árvore com até $6 \mathrm{~m}$ de altura, nativa do Brasil. É uma planta pioneira, rústica, perene e heliófita sendo adequada para uso em arborização urbana e recuperação de áreas degradadas (LORENZI, 2008). De acordo com Carvalho (2007), esta espécie é importante fonte melífera, sendo uma das poucas espécies arbóreas melíferas de inverno. Produz lenha de baixo valor e sua madeira é utilizada para cabos de ferramentas ou de utensílios domésticos.

Bougainvillea glabra Choisy. (Nyctaginaceae): planta espinescente nativa do Brasil, com altura de até $20 \mathrm{~m}$, dotada de copa alongada e densa. Com folhas membranáceas, perenifólia e heliófita, é uma planta pioneira de rápido crescimento (LORENZI, 2008).

\subsection{Obtenção e análise de dados}

O local de coleta foi o Campus III da Universidade Federal do Paraná. Para realização do experimento foram coletados, em toda a área externa da copa, folhas e ramos vivos com diâmetro inferior a $0,7 \mathrm{~cm}$, considerados por Schroeder; Buck (1970) como combustível fino, com tempo de resposta de 1 hora (1h timelag) em relação à variação ambiental. Quando em 
estado seco, este material é categorizado como perigoso, por apresentar menor temperatura de ignição, facilitar a ignição e acelerar a propagação do fogo (SOARES et al., 2017).

Ainda em campo, foi obtida a massa do material combustível úmido. Posteriormente este material foi encaminhado ao laboratório para secagem em estufa, por um período de 48 horas, a $75^{\circ} \mathrm{C}$. O teor de umidade foi calculado por meio da equação 1, apresentada por Soares et al. (2017). A obtenção das massas foi realizada com o emprego de um dinamômetro (graduado em grama).

$$
T U=\left(\frac{\mathrm{Mu}-\mathrm{Ms}}{\mathrm{Ms}}\right) * 100
$$

(Equação 1)

em que: $T U=$ teor de umidade do material combustível, em $\% ; M u=$ massa úmida do material combustível, no momento da coleta, em g; $M s=$ massa seca do material combustível, após secagem em estufa, em $\mathrm{g}$.

O material seco em estufa foi transportado em sacos vedados até o local de execução das queimas controladas, que se caracteriza por: terreno plano, cobertura e barreiras contra o vento. As queimas foram executadas de acordo com o esquema demonstrado na Figura 1. Para cada queima utilizou-se uma carga de $1 \mathrm{~kg}$ de material combustível seco (com teor de umidade máximo de 12\%), distribuído homogeneamente em parcela de $1 \mathrm{~m}^{2}(1 \times 1 \mathrm{~m})$. Todas as queimas foram filmadas para posterior análise da altura de chama e da velocidade de propagação do fogo.

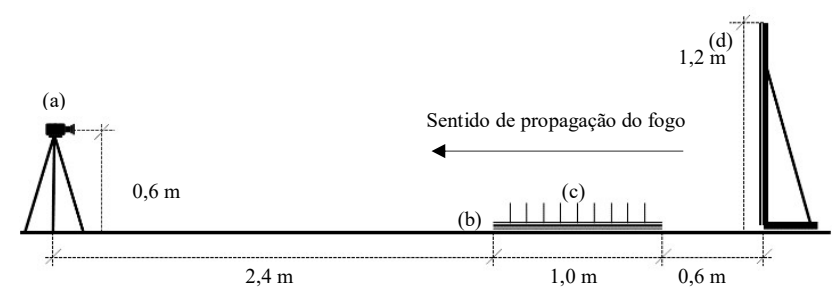

Figura 1. Queima controlada: vista lateral. Nota: (a) câmera; (b) parcela com material combustível; (c) régua para auxílio da determinação da velocidade propagação do fogo; (d) painel para leitura da altura de chama.

Figure 1. Controlled burn: side view.

Foram realizadas, para cada espécie, 10 repetições de queima. Para fins comparativos, as queimas das espécies selecionadas foram alternadas com queimas de $P$. taeda, tornando-as queimas pareadas, visando a semelhança das condições meteorológicas de temperatura e umidade relativa. Estas variáveis foram obtidas antes e após cada queima por meio de uma miniestação meteorológica.

Para cálculo da densidade da manta de material combustível $\left(\mathrm{kg} \cdot \mathrm{m}^{-3}\right)$, foi obtida da altura em cada um dos lados da parcela, com auxílio de uma régua, e realizada a média. A altura de chama foi obtida a cada $10 \mathrm{~cm}$ de propagação do fogo, tendo como referência uma régua graduada, sendo realizadas oito observações por queima (totalizando 80 leituras por espécie).

A velocidade de propagação, em $\mathrm{cm} \cdot \mathrm{s}^{-1}$, foi determinada por meio da relação entre distâncias pré-estabelecidas e o tempo gasto pela linha da frente de fogo para percorrê-las, sendo que, para cada repetição, foram obtidas oito tomadas de tempo, totalizando desta forma, 80 leituras por espécie.

O material residual foi coletado na área central da parcela de queima, com auxílio de um gabarito de $400 \mathrm{~cm}^{2}(20 \times 20$ $\mathrm{cm}$ ), para então determinar a quantidade de material consumido a partir da Equação 2.

$$
\mathrm{Mc}=\left(\frac{1000-\mathrm{Rq}}{10}\right)
$$

em que: $\mathrm{Mc}=$ quantidade de material consumido pelo fogo durante a combustão, em \%; Rq = resíduo deixado após a queima, em g.m-2.

A intensidade do fogo foi obtida por meio da fórmula de Byram (1959) (Equação 3).

$$
\mathrm{I}=\mathrm{H} . \mathrm{w} \cdot \mathrm{r}
$$

em que: $\mathrm{I}=$ intensidade do fogo, em kcal. $\mathrm{m}^{-1} \cdot \mathrm{s}^{-1} ; \mathrm{H}=$ poder calorífico, em kcal.kg-1 $\left( \pm 4.000 \mathrm{kcal} . \mathrm{kg}^{-1}\right) ; \mathrm{w}=$ massa do combustível disponível por área, em kg. $\mathrm{m}^{-2} ; \mathrm{r}=$ velocidade de propagação do fogo, em m.s. ${ }^{-1}$.

Utilizou-se o valor de Poder Calorífico superior (PCS) conhecido de P. taeda $\left(4701,0 \mathrm{kcal} . \mathrm{kg}^{-1}\right)$, M. grandiflora (4625,5 kcal. $\left.\mathrm{kg}^{-1}\right)$, M. champaca (4319,0 kcal.kg-1), C. sylvestris $\left(4805,0 \quad \mathrm{kcal}_{\mathrm{kg}}^{-1}\right), \quad V$. odoratissimum $(4619,5$ kcal. $\mathrm{kg}^{-1}$ ) e $B$. glabra (4162,0 kcal. $\mathrm{kg}^{-1}$ ) (ASSUNÇÃO, 2013). De acordo com Soares et al. (2017), o poder calorífico de combustíveis florestais é alta e não varia de maneira significativa, correspondendo a um valor aproximado de 4000,0 kcal. $\mathrm{kg}^{-1}$.

Com os dados experimentais obtidos, procedeu-se à análise estatística das variáveis por meio da análise de variância (ANOVA), do teste de comparação de médias, pelo método Student-Newman-Keuls (SNK) e análise de conglomerados por meio do método Ward, Euclidiana quadrada, considerando a média das variáveis: altura de chama, velocidade de propagação, material consumido e intensidade do fogo.

\section{RESULTADOS}

O teor de umidade do material combustível das espécies está apresentado na Tabela 1 .

Tabela 1. Teor médio de umidade do material combustível das espécies analisadas.

Table 1. Average fuel moisture content of the analyzed species.

\begin{tabular}{l|c|c|c|c}
\hline \multirow{2}{*}{ Nome científico } & Rep. & $\begin{array}{c}\text { Massa } \\
\text { úmida (g) }\end{array}$ & $\begin{array}{c}\text { Massa } \\
\text { seca (g) }\end{array}$ & $\begin{array}{c}\text { Teor de } \\
\text { umidade } \\
(\%)\end{array}$ \\
\hline M. grandiflora & 10 & 2363,9 & 1096,4 & $116,9 \mathrm{a}$ \\
M. champaca & 10 & 3054,1 & 1089,6 & $183,7 \mathrm{~b}$ \\
C. sylvestris & 10 & 3103,0 & 1181,0 & $164,6 \mathrm{~b}$ \\
V. odoratissimum & 10 & 3501,0 & 1331,0 & $163,1 \mathrm{~b}$ \\
B. glabra & 10 & 4435,0 & 1457,0 & $210,6 \mathrm{c}$ \\
\hline
\end{tabular}

Nota: Rep: número de repetições.

A espécie que apresentou maior teor de umidade foi $B$. glabra $(210,6 \%)$, seguida de $M$. champaca (183,7\%), enquanto a menor foi $M$. grandiflora $(116,9 \%)$.

Os valores médios dos parâmetros do comportamento do fogo (altura de chama, velocidade de propagação, material consumido e intensidade do fogo) e das variáveis meteorológicas (temperatura do ar, umidade relativa do ar e velocidade do vento) registradas durante os ensaios, estão apresentados na Tabela 2 . 
Tabela 2. Comparação de médias dos parâmetros do comportamento do fogo e as médias das variáveis meteorológicas registradas durante os ensaios.

Table 2. Comparison of averages of the fire behavior parameters and averages of weather variables recorded during the experiments.

\begin{tabular}{|c|c|c|c|c|c|c|c|c|c|}
\hline \multirow[b]{2}{*}{ Código } & \multirow[b]{2}{*}{ Nome científico } & \multirow[b]{2}{*}{$\begin{array}{l}\text { Densidade } \\
\text { da manta } \\
\left(\mathrm{kg} \cdot \mathrm{m}^{-3}\right)\end{array}$} & \multicolumn{4}{|c|}{ Parâmetros do comportamento do fogo } & \multicolumn{3}{|c|}{ Variáveis meteorológicas } \\
\hline & & & $\begin{array}{c}\text { Altura } \\
\text { de chama } \\
(\mathrm{cm})\end{array}$ & $\begin{array}{c}\text { Velocidade de } \\
\text { propagação } \\
\left(\mathrm{cm} \cdot \mathrm{s}^{-1}\right)\end{array}$ & $\begin{array}{c}\text { Material } \\
\text { consumido } \\
(\%)\end{array}$ & $\begin{array}{c}\text { Intensidade } \\
\text { do fogo } \\
\left(\mathrm{kcal} \cdot \mathrm{m}^{-1} \cdot \mathrm{s}^{-1}\right)\end{array}$ & $\begin{array}{c}\mathrm{T} \\
\left({ }^{\circ} \mathrm{C}\right)\end{array}$ & $\begin{array}{l}\text { UR } \\
(\%)\end{array}$ & $\begin{array}{l}\text { V. vento } \\
\left(\mathrm{m} \cdot \mathrm{s}^{-1}\right)\end{array}$ \\
\hline 1 & M. grandiflora & $11,86 \mathrm{ab}$ & $40,20 \mathrm{f}$ & $0,74 \mathrm{bc}$ & $86,22 \mathrm{de}$ & $34,37 \mathrm{bc}$ & \multirow{2}{*}{24,5} & \multirow{2}{*}{53,2} & \multirow{2}{*}{0,0} \\
\hline 1.p & P. taeda & $13,95 \mathrm{bc}$ & $49,89 \mathrm{~g}$ & 0,93 cde & 84,60 cde & $43,72 \mathrm{~cd}$ & & & \\
\hline 2 & M. champaca & $7,44 \mathrm{a}$ & 39,47 ef & $1,17 \mathrm{f}$ & 78,10 cde & $50,40 \mathrm{~d}$ & \multirow{2}{*}{21,0} & \multirow{2}{*}{62,5} & \multirow{2}{*}{0,0} \\
\hline 2.p & P. taeda & $12,52 \mathrm{~b}$ & $33,03 \mathrm{~cd}$ & 0,89 cde & $72,50 \mathrm{bc}$ & $41,98 \mathrm{~cd}$ & & & \\
\hline 3 & C. sylvestris & 23,85 ef & $23,76 \mathrm{~b}$ & $0,54 \mathrm{~b}$ & $60,43 \mathrm{~b}$ & $25,95 \mathrm{~b}$ & \multirow{2}{*}{15,3} & \multirow{2}{*}{61,1} & \multirow{2}{*}{0,0} \\
\hline 3.p & P. taeda & $20,06 \mathrm{def}$ & $33,24 \mathrm{~cd}$ & $1,05 \mathrm{ef}$ & $89,96 \mathrm{e}$ & $49,17 \mathrm{~d}$ & & & \\
\hline 4 & V. odoratissimum & $17,80 \mathrm{~cd}$ & $28,14 b c$ & $0,81 \mathrm{~cd}$ & 79,91 cde & $37,56 \mathrm{c}$ & \multirow[b]{2}{*}{19,4} & \multirow[b]{2}{*}{55,9} & \multirow[b]{2}{*}{0,0} \\
\hline 4.p & P. taeda & $19,68 \mathrm{de}$ & $34,48 \mathrm{de}$ & $1,00 \mathrm{def}$ & $89,96 \mathrm{e}$ & $40,59 \mathrm{~cd}$ & & & \\
\hline 5 & B. glabra & $24,67 \mathrm{f}$ & $10,65 \mathrm{a}$ & $0,19 \mathrm{a}$ & $29,12 \mathrm{a}$ & $7,78 \mathrm{a}$ & \multirow{2}{*}{20,7} & \multirow{2}{*}{66,2} & \multirow{2}{*}{0,0} \\
\hline 5.p & P. taeda & $13,33 \mathrm{bc}$ & $41,69 \mathrm{f}$ & 0,87 cde & $72,87 \mathrm{bcd}$ & $41,08 \mathrm{~cd}$ & & & \\
\hline
\end{tabular}

Nota: Médias com letras diferentes apresentam diferenças estatísticas entre si pelo teste de comparação de médias SNK a 95,0\% de probabilidade; para cada espécie analisada foram realizadas queimas de $P$. taeda, codificadas com o número referente a esta espécie, seguidas pela letra p (pareadas) para a comparação dos resultados entre estas.

A maior densidade foi observada na manta constituída por material combustível de B. glabra (5) $\left(24,67 \mathrm{~kg} \cdot \mathrm{m}^{-3}\right)$, ou seja, o arranjo deste material ficou mais compactado que os demais, e a manta que apresentou menor densidade foi de $M$. chamapaca (2), com 7,44 kg.m $\mathrm{m}^{-3}$.

A espécie M. champaca (2) apresentou altura de chama de $39,47 \mathrm{~cm}$, sendo a única espécie a demostrar um valor médio da altura de chama superior em relação a $P$. taeda (2.p), que apresentou altura de chama de 33,03 cm. M. grandiflora (1) apresentou altura de chama de $40,2 \mathrm{~cm}$, porém foi inferior e estatisticamente diferente de $P$. taeda (1.p) $(49,89 \mathrm{~cm})$. As demais espécies indicaram uma altura de chama inferior e com diferenças estatísticas, quando comparadas às respectivas queimas de $P$. taeda. Dentre todas as espécies analisadas, as que se destacaram por apresentarem os menores valores de altura de chama foram $C$. sylvestris (3), com altura de chama média de 23,76 cm, e B. glabra (5) com altura de chama média de $10,65 \mathrm{~cm}$.

Com relação a velocidade de propagação da frente de chama, as espécies que apresentaram menor valor foram $B$. glabra (5) $\left(0,19 \mathrm{~cm}^{-1}\right)$ e C. sylvestris (3) $\left(0,54 \mathrm{~cm} \cdot \mathrm{s}^{-1}\right)$, enquanto $M$. champaca (2) apresentou a maior velocidade de propagação, $1,17 \mathrm{~cm} \cdot \mathrm{s}^{-1}$. M. grandiflora (1) e $V$. odoratissimum (4) não apresentaram diferença estatística em relação aos valores apresentados pelas respectivas queimas de $P$. taeda. A velocidade de propagação da chama de $P$. taeda variou de 0,87 a $1,05 \mathrm{~cm} \cdot \mathrm{s}^{-1}$.

A espécie com menor percentual de material combustível consumido foi B. glabra (5) (29,12\%), seguida de C. sylvestris (3) $(60,43 \%)$. As demais espécies não demonstraram diferença estatística quando comparadas as respectivas queimas de $P$. taeda. Para $P$. taeda a intensidade do fogo variou de 40,59 a $49,17 \mathrm{kcal} \cdot \mathrm{m}^{-1} \cdot \mathrm{s}^{-1}$. A espécie que apresentou menor intensidade do fogo foi $B$. glabra (5) $\left(7,78 \mathrm{kcal} . \mathrm{m}^{-1} \cdot \mathrm{s}^{-1}\right)$, seguida de $C$. sylvestris (3) $\left(25,95 \mathrm{kcal} \cdot \mathrm{m}^{-1} \cdot \mathrm{s}^{-1}\right)$, enquanto $M$. champaca (2) demonstrou ser a espécie com maior intensidade dentre as estudadas e não apresentou diferença estatística em relação a P. taeda (2.p).

$\mathrm{O}$ dendrograma referente às espécies analisadas é apresentado na Figura 2. Com a aplicação da análise de agrupamento, realizada com os parâmetros de comportamento do fogo, classificou a combustibilidade das cinco espécies avaliadas em dois grupos: a) alta combustibilidade: $M$. grandiflora, $V$. odoratissimum e $M$. champaca; e b) baixa combustibilidade: B. glabra e C. sylvestris.

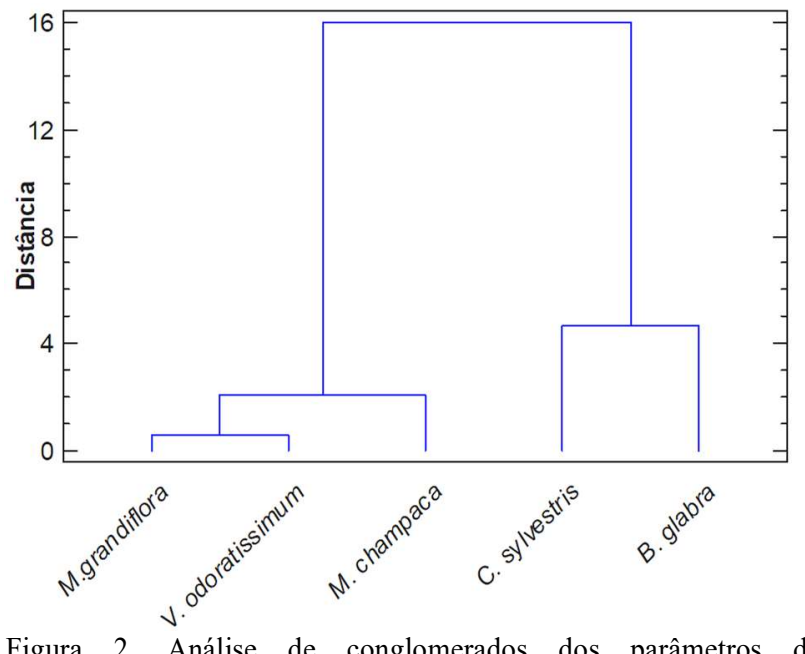

Figura 2. Análise de conglomerados dos parâmetros do comportamento do fogo das espécies selecionadas.

Figure 2. Analysis of conglomerates of the fire behavior parameters from the selected species.

\section{DISCUSSÃO}

B. glabra foi a espécie que apresentou menores valores para os parâmetros de comportamento do fogo e maior teor de umidade, sendo este descrito por diversos pesquisadores como um fator chave que afeta a inflamabilidade (WHITE; ZIPPERER, 2010). Esses resultados corroboram com Kovalsyki et al. (2016), que apontam a espécie como pouco inflamável.

Outra característica observada para $B$. glabra e $C$. sylvestris foi a densa manta formada pela biomassa, entretanto este fator não foi verificado em campo. A densidade da serapilheira é fortemente correlacionada com a velocidade de propagação, a altura de chama e a duração da combustão (CLARKE et al., 2014), uma vez que influi na quantidade de oxigênio disponível para a combustão (SOARES et al., 2017). Assim, observou-se que as espécies que tiveram valores inferiores de combustibilidade, formaram mantas mais compactadas, como também evidenciado por Scarff; Westoby (2006). 
De acordo com Wyse et al., (2016) para as espécies com grau de inflamabilidade mais elevado, ocorre um alto consumo de biomassa, características estas observadas para $M$. grandiflora, M. champaca e V. odoratissimum.

$V$. odoratissimum apresentou combustibilidade similar a $P$. taeda (4.p), porém sua inflamabilidade é moderada (BATISTA et al., 2012) e, devido a sua resiliência, vem sendo aplicada na prevenção de incêndios florestais na China, local de onde é originária (ZHANG et al., 2017).

Batista et al. (2012), observaram que o material fresco de $M$. grandiflora e $C$. sylvestris apresentam baixo tempo de ignição, classificando-as como altamente inflamáveis. No presente estudo, M. grandiflora indicou combustibilidade similar a $P$. taeda, entretanto $C$. sylvestris apresentou resultados satisfatórios, assim, sugere-se análise de características foliares, uma vez que estas também podem influenciar a inflamabilidade (WHITE; ZIPPERER, 2010; CURT et al., 2011).

De acordo com a literatura, $M$. grandiflora, $M$. champaca, $V$. odoratissimum e $C$. sylvestris apresentam óleos essenciais em suas folhas. Plantas medicinais e aromáticas, coníferas e Eucalyptus spp. são capazes de armazenar isoprenoides voláteis em reservatórios e compartimentos especializados (CICCIOLI et al., 2014), os quais podem aumentar a inflamabilidade, em especial no que diz respeito ao tempo de ignição (ALESSIO et al. 2008). Entretanto, se a espécie conter alto teor de umidade, o vapor d'água pode diluir estes combustíveis gasosos liberados, levando a um prolongado período de ignição (KAUF et al., 2015). Este efeito também pode ocorrer em função da espessura da cutícula das folhas, uma vez que cutículas mais espessas dificultam a vaporização da água e de qualquer óleo (MURRAY et al., 2013).

Outro fator a ser considerado é a variação das características de inflamabilidade conforme as condições meteorológicas. Conforme Alessio et al. (2008), pode haver sazonalidade na temperatura de pirólise e no tempo de ignição de acordo com as estações do ano, uma vez que há alteração na disponibilidade de água. Deste modo, também pode ocorrer a variação do nível de inflamabilidade de uma determinada espécie em função da localização (WHITE; ZIPPERER, 2010).

Listas de espécies potenciais para uso em cortinas de segurança têm sido desenvolvidas, entretanto estas são baseadas em informações empíricas, ou em fatores como resistência a seca por exemplo, sem padronização e para locais específicos (e.g. lista de espécies elaborada para Nova Zelândia (SCION, 2018)), portanto devem ser utilizadas com cautela (WHITE; ZIPPERER, 2010), uma vez que espécies mais inflamáveis podem determinar a inflamabilidade geral de uma combinação (WYSE et al., 2018).

Segundo Murray et al. (2018) as pesquisas para identificar amplas generalidades nas características morfológicas, químicas e estruturais de espécies de baixa inflamabilidade ainda estão no estágio pioneiro.

Além de avaliar as características de combustibilidade e inflamabilidade de forma segregada e individual, recomendase realizar testes em campo para avaliar respostas considerando a arquitetura inteira das plantas e serapilheira, como também conjuntos de espécies (BURGER; BOND, 2015; WYSE et al., 2018).

\section{CONCLUSÕES}

As espécies B. glabra e $C$. sylvestris apresentaram melhores resultados em relação às variáveis do fogo (baixa combustibilidade), indicando maior potencial para a utilização em cortinas de segurança entre as espécies analisadas.

M. grandiflora, M. champaca e $V$. odoratissimum apresentaram combustibilidade superior ou similares a $P$. taeda sendo, entre as espécies estudadas, as menos indicadas para uso em cortina de segurança.

As variáveis analisadas forneceram importante base de dados sobre o comportamento do fogo das espécies estudadas.

Recomenda-se a investigação de parâmetros de inflamabilidade, análise de características foliares, sazonalidade e testes em campo em estudos futuros, a fim de corroborar com os resultados deste trabalho para a indicação de uso de espécies em cortinas de segurança na prevenção de incêndios florestais.

\section{REFERÊNCIAS}

ALESSIO, G. A.; PEÑUELAS, J.; LLUSIÀ, J.; OGAYA, R.; ESTIARTE, M.; DE LILLIS, M. Influence of water and terpenes on flammability in some dominant Mediterranean species. International Journal of Wildland Fire, Rosyn, v. $17, \quad$ n. 2 , p. 274-286, 2008. DOI: https://dx.doi.org/10.1071/WF07038

ASSUNÇÃO, R. Determinação do potencial energético de espécies arbóreas e arbustivas para 0 uso potencial em cortina de segurança. Curitiba: UFPR, 2013. (Relatório técnico).

BATISTA, A. C.; BIONDI, D.; TETTO, A. F.; ASSUNÇÃO, R.; TRES, A.; TRAVENISK, R. C. C.; KOVALSYKI, B. Evaluation of the flammability of trees and shrubs used in the implementation of green barriers in southern Brazil. General Technical Report PSW-GTR, v. 245, p. 256264, 2012. Disponível em: $<$ https://www.fs.fed.us/psw/publications/documents/psw gtr245/psw_gtr245_256.pdf $>$. Acesso em: 08 ago. 2017.

BATISTA, A. C.; BIONDI. D. Avaliação da inflamabilidade de Ligustrum lucidum Aiton (Oleaceae) para uso potencial em cortinas de segurança na região sul do Brasil. Revista Brasileira de Ciências Agrárias, Recife, v. 4, n. 4, p. 435 439, 2009. DOI: http://dx.doi.org/10.5039/agraria.v4i4a11

BATISTA, A. C.; TETTO, A. F.; DEPPE, F.; GRODZKI, L.; GRASSI, J. T. Análise dos impactos das mudanças climáticas sobre o risco de incêndios florestais no estado do Paraná. Scientia forestalis, Piracicaba, v. 42, n. 104, p. 491-501, 2014.

BIANCHETTI, A.; ZANON, A.; FOWLER, J. A. P. Substrato e temperatura para germinação das sementes de magnólia-amarela (Michelia champaca Linn). Amapá: Embrapa, 2000. 2 p. (Comunicado Técnico). Disponível em: $<$ https://www.embrapa.br/busca-de-publicacoes//publicacao/345883/substratos-e-temperaturas-para-agerminacao-de-sementes-de-magnolia-amarela-micheliachampaca-linn>. Acesso em: 15 nov. 2017.

BIONDI, D.; BATISTA, A. C.; MARTINI, A. The flammability of ornamental species with potential for use in highways and wildland urban interface (WUI) in Southern Brazil. In: VIEGAS, D. M (Ed.). Advances in forest fire research. Coimbra: Imprensa da Universidade de Coimbra, 2014. p. 992-997. 
BOVIO, G.; MARCHETTI, M.; TORNARELLI, L.; SALIS, M.; VACCHIANO, G.; LOVREGLIO, R.; ELIA, M.; FIORUCCI, P.; ASCOLI, D. Forest fires are changing: let's change the fire management strategy. Forest@, Bari, Itália, v. 14, 2017. p. 202-205. DOI: https://dx.doi.org/10.3832/efor2537-014

BOWMAN, D. M. J. S.; BALCH, J.; ARTAXO, P.; BOND, W. J.; COCHRANE, M. A.; D'ANTONIO, C. M.; DEFRIES, R.; JOHNSTON, F. H.; KEELEY, J. E.; KRAWCHUK, M. A.; KULL, C. A.; MACK, M.; MORITZ, M. A.; PYNE, S.; ROOS, C. I.; SCOTT, A. C.; SODHI, N. S.; SWETNAM, T. W. The human dimensiono f fire regimes on Earth. Journal of Biogeography, Oxford, v. 38, n. 12 , p. 2223-2236, 2011. DOI: https://dx.doi.org/10.1111/j.1365-2699.2011.02595.x

BURGER, N.; BOND, W. J. Flammability traits of Cape shrubland species with different post-fire recruitment strategies. South African Journal of Botany, Pretoria, v. 101, p. 40-48, 2015. DOI https://dx.doi.org/10.1016/j.sajb.2015.05.026

BYRAM, G. M. Combustion of forest fuels. In: DAVIS, K. P. (Coor.). Forest Fire: control and use. New York: Mc Graw Hill, 1959. p. 77-84. Disponível em: < https://www.frames.gov/catalog/9652>. Acesso em: 10 nov. 2017.

CARVALHO, P. E. R. Cafezeiro-do-mato: Casearia sylvestris. Colombo: Embrapa Florestas, 2007. 16 p. (Circular Técnica, 138). Disponível em: $<$ https://www.embrapa.br/busca-de-publicacoes//publicacao/313896/cafezeiro-do-mato---caseariasylvestris>. Acesso em: 12 nov. 2017.

CICCIOLI, P.; CENTRITTO, M.; LORETO, F. Biogenic volatile organic compound emissions from vegetation fires. Plant, Cell \& Environment, Oxford, v. 37, n. 8, p. 1810-1825, 2014.2 DOI https://dx.doi.org/10.1111/pce.12336

CLARKE, P. J.; PRIOR, L. D.; FRENCH, B. J.; VINCENT, B.; KNOX, K. J. E.; BOWMAN, D. M. J. S. Using a rainforest-flame forest mosaic to test the hypothesis that leaf and litter fuel flammability is under natural selection. Oecologia, Berlin, v. 176, n. 4, p. 1123-1133, 2014. DOI: https://dx.doi.org/10.1007/s00442-014-3071-y

CORDELL, S. Greenstrips in Hawaii - can they protect high-value ecosystems from wildfire?. 2017. Disponível em: $<$ http://www.pacificfireexchange.org $>$. Acesso em: 04 ago. 2018.

CURRAN, T. J.; PERRY, G. L. W.; ALAM, M. A. Managing fire and biodiversity in the wildland-urban interface: a role for green firebreaks. Fire, Basel, v. 1, n. 3, p. 1-3, 2017. DOI: https://dx.doi.org/10.3390/fire1010003

CURT, T.; SCHAFFHAUSER, A.; BORGNIET, L.; DUMAS, C.; ESTÈVES, R.; GANTEAUME, A.; JAPPIOT, M.; MARTIN, W.; N'DIAYE, A.; POILVET, B. Litter flammability in oak woodlands and shrublands of southeastern France. Forest Ecology and Management Amsterdam, v. 261, n. 12, p. 2214-2222, 2011. DOI: https://dx.doi.org/10.1016/j.foreco.2010.12.002

GILMAN, E. F.; WATSON, D. G. Magnolia grandiflora: southern magnolia. Florida: University of Florida, 2011a 3 p. Disponível em: <http://edis.ifas.ufl.edu/st371>. Acesso em: 29 out. 2017

GILMAN, E. F.; WATSON, D. G. Viburnum odoratissimum: sweet viburnum. Florida: University of Florida, 2011b. 3 p. Disponível em: <http://edis.ifas.ufl.edu/st659>. Acesso em: 29 out. 2017.

HALTENHOFF, H. Silvicultura preventiva. 2. ed. Santiago: Ministerio de Agricultura, Corporación Nacional Forestal, 2006. 40 p. (Manual técnico, 452)

HO, T. L.; BATISTA, A. C.; TETTO, A. C. Forest fire risk forecast for the state of Santa Catarina due to climate changes. Floresta, Curitiba, v. 41, n. 4, p. 427-436, 2017. DOI: http://dx.doi.org/10.5380/rf.v47i4.50877

KAUF, Z.; FANGMEIER, A.; ROSAVEC, R.; SPANJOL, Z. Seasonal and local differences in leaf litter flammability of six Mediterranean tree species. Environmental Management, Nova York, v. 55, n. 3, p. 687-701, 2015. DOI: https://dx.doi.org/10.1007/s00267-014-0427-3

KOVALSYKI, B.; TAKASHINA, I. K.; TRES, A.; TETTO, A. F.; BATISTA, A. C. Inflamabilidade de espécies arbóreas para uso em cortina de segurança na prevenção de incêndios florestais. Pesquisa Florestal Brasileira, Colombo, v. 36, n. 88, p. 387-391, 2016. DOI: http://dx.doi.org/10.4336/2016.pfb.36.88.991

LORENZI, H. Árvores brasileiras: manual de identificação e cultivo de plantas arbóreas nativas do Brasil. 5. ed. Nova Odessa: Instituto Plantarum, 2008.

LORENZI, H.; SOUZA, H. M.; TORRES, M. A.; BACHER, L. B. Árvores exóticas no Brasil: madeireiras, ornamentais e aromáticas. Nova Odessa: Instituto Plantarum, 2003. 384 p.

MAPA MINISTÉRIO DA AGRICULTURA, PECUÁRIA E ABĀSTECIMENTO. Escolha das espécies utilizadas como quebra-vento. 2016. Disponível em: $<$ http://www.agricultura.gov.br>. Acesso em: 06 ago. 2018.

MURRAY, B. R.; HARDSTAFF, L. K.; PHILLIPS, M. L. Differences in leaf flammability, leaf traits and flammability-trair relationships between native and exotic plants species of dry sclerophyll forest. Plos one, San Francisco, v. 8, n. 11, p. 1-8, 2013. DOI: https://dx.doi.org/10.1371/journal.pone.0079205

MURRAY, B. R.; MARTIN, L. J.; BROWN, C.; KRIX, D. W.; PHILLIPS, M. L. Selecting low-flammability plants as green firebreaks within sustainable urban garden design, Fire, v. 1, n. 1, 4 p., 2018. Doi: https://dx.doi.org/10.3390/fire1010015

OLIVEIRA, D. S. F.; FRANCHIN, A. G.; MARÇAL JUNIOR, O. Disponibilidade de frutos de Michelia champaca L. (Magnoliaceae) e seu consumo por aves na área urbana de Uberlândia, MG. Bioscience jornal, Uberlândia, v. 29, n. 6, p. 2053-2065, 2013

OUTCALT, K. W. Magnolia grandiflora. In: BURNS, R. M.; HONKALA, B. H. Silvics of North America: hardwoods. 2. vol. Washington: Forest Service, 1990. Disponível em: $<$ https://www.srs.fs.usda.gov/pubs/misc/ag_654_vol2.pdf >. Acesso em: 29 out. 2017

SCARFF, F. R.; WESTOBY, M. Leaf litter flammability in some semi-arid Australian woodlands. Functional Ecology, Oxford, v. 20, n. 5, p. 745-752, 2006.

SCHROEDER, M. J.; BUCK, C. C. Fire weather. Washington, DC: USDA Forest Service, 1970.

SCION. Flammability of native plant species. 2018. Disponível em: $<$ http://www.ruralfireresearch.co.nz/_data/assets/pdf_file /0007/64168/31328-Flammability-Brochure.pdf $>$. Acesso em: 06 ago. 2018. 
SEIDL, R.; THOM, D.; KAUTZ, M.; MARTIN BENITO, D.; PELTONIEMI, M.; VACCHIANO, G.; WILD, J.; ASCOLI, D.; PETR, M.; HONKANIEMI, J.; LEXER, M. J.; TROTSIUK, V.; MAIROTA, P.; SVOBODA, M.; FABRIKA, M.; NAGEL, T. A.; REYER, C. P. O. Forest disturbances under climate change. Nature Climate Change, v. 7, n. 6, p. 395-402, 2017. DOI: https://dx.doi.org/10.1038/nclimate3303

SOARES, R. V.; BATISTA, A. C.; TETTO, A. F. Incêndios florestais: controle, efeitos e uso do fogo. 2. ed. Curitiba: Editores independentes, 2017. 255 p.

TIAN, X.; SHU, L.; WANG, M. Study on eight tree species combustibility and fuel break effectiveness. In: CONFERENCIA INTERNACIONAL SOBRE INCENDIOS FORESTALES, 4., 2007, Sevilla: Acta... Sevilla: Ministerio de Meio Ambiente, 2007. p. 1-11. Disponível em: $<$ http://www.fire.uni-freiburg.de/sevilla2007/contributions/html/es/autor T.html>. Acesso em: 03 dez. 2017.

VÉLEZ, R. Preventing forest fires through silviculture. Unasylva: FAO, 2009. Disponível em: $<$ http://www.fao.org/docrep/t9500e/t9500e03.htm>. Acesso em: 03 dez. 2017.

WADE, G. L. Growing southern magnolia. University of Georgia, 2012. Disponível em: $<$ http://extension.uga.edu/publications/detail.cfm?number $=\mathrm{c} 974>$. Acesso em: 29 out. 2017.
WHITE, R. H.; ZIPPERER, W. C. Testing and classification of individual plants for fire behaviour: plant selection for the wildland-urban interface. International Journal of Wildland Fire, Rosyn, v. 19, n. 2, p. 213-227, 2010. DOI: https://dx.doi.org/10.1071/WF07128

WYSE, S. V.; PERRY, G. L. W.; CURRAN, T. J. Shoot-level flammability of species mixtures is driven by the most flammable species: implications for vegetation-fire feedbacks favouring invasive species. Ecosystems, New York, v. 21, n. 5, p. 886-900, 2018. DOI: https://dx.doi.org/10.1007/s10021-017-0195-z

WYSE, S. V.; PERRY, G. L. W.; O'CONNELL, D. M.; HOLLAND, P. S.; WRIGHT, M. J.; HOSTED, C. L.; WHITELOCK, S. L.; GEARY, I. J.; MAURIN, K. J. L.; CURRAN, T. J. A quantitative assessment of shoot flammability for 60 tree and shrub species supports rankings based on expert opinion. International Journal of Wildland Fire, Rosyn, v. 25, n. 4, p. 466-477, 2016. DOI: https://dx.doi.org/10.1071/WF15047

ZHANG, Y.; FU, T. T.; HUANG, C. L.; CHENG, D. M.; HUANG, R. L.; ZHANG, Z. X. Insecticidal activity of the soil in the rhizosphere of Viburnum odoratissimum against Solenopsis invicta (Hymenoptera: Formicidae). Sociobiology, Chico, v. 64, n. 1, p. 1-6, 2017. DOI: https://dx.doi.org/10.13102/sociobiology.v64i1.1067 